\title{
Transplantation of mesenchymal stem cells in a laryngeal carcinoma patient with radiation myelitis
}

\author{
Jun Liang ${ }^{*}$, Fan Wang, Dandan Wang, Huayong Zhang, Cheng Zhao, Shiying Wang and Lingyun Sun
}

\begin{abstract}
Radiation myelitis is a rather rare but devastating complication following therapeutic irradiation to neoplasms when the spinal cord is included within the radiation field. Symptoms of radiation myelitis with the therapeutic doses of radiation commonly employed are usually delayed and most often appear about 6 to 24 months following irradiation. So far, no treatment has proved satisfactory.

Transplantation of allogeneic mesenchymal stem cells has been a promising therapy strategy for many disorders in the central nervous system, such as multiple sclerosis, neuromyelitis optica, and autoimmune encephalomyelitis. The cell-base therapy has shown to act to limit inflammation of central nervous system, stimulate neurogenesis, protect axons and promote remyelination. But it has not been established as a therapeutic option for radiation myelitis. In this report, we describe the outcome of allogeneic umbilical cord-derived mesenchymal stem cell transplantation in a patient with laryngeal carcinoma who developed radiation-induced myelitis of his spinal cord with characteristic magnetic resonance imaging changes.
\end{abstract}

\section{Introduction}

Radiation myelitis, while rare, is one of the gravest complications in radiation therapy. Some reports have attempted to decrease the incidence of radiation myelitis by minimizing the total radiation dose and the fraction size $[1,2]$. However, few articles have focused on the therapy of radiation myelitis. Up to now, therefore, the treatment of radiation myelitis consists mainly of its prevention; and when this fails, symptomatic measures are usually employed. Some cases demonstrated dramatic response to high-dose steroid treatment $[3,4]$, but steroids were shown to obtain temporary remissions or only delay the progression of radiation myelitis for a short period of time [5]. Furthermore, other cases showed that steroids had no influence on the progression of disease [6].

Mesenchymal stem cells (MSCs), a subset of nonhematopoietic cells, possess pluripotent features and can be obtained from different sources such as bone marrow, umbilical cord (UC), UC blood, and adipose tissue of the human body. These cells are able to differentiate into

\footnotetext{
* Correspondence: liangjunlzk@hotmail.com

Department of Rheumatology and Immunology, the Affiliated Drum Tower Hospital of Nanjing University Medical School, 321 Zhongshan Road, Nanjing, CN 210008, P. R. China
}

tissues of mesenchymal lineages, including bone, cartilage, fat, tendon, muscle, and stroma [7-11]. Recently, research has shown that MSCs can differentiate into neural stem cells, mature functional neurons, and glial cells [12-15], while no data suggest that MSCs can differentiate into axons. Liu et al. [16], however, have reported that acellular spinal cord scaffold seeded with MSCs is able to bridge a spinal cord cavity and promote ong-distance axon regeneration and functional recovery in spinal cord injury rats. The second property of MSCs is their capacity for immunomodulation. Emerging has demonstrated that human MSCs can hibit, in a dose-dependent manner, the proliferation d cytokine production of many allogeneic immune , such as T cells, B cells, natural killer (NK) cells, dendritic cells [17-19]. MSCs have also been found inved in MSC-mediated immunoregulation, such (IL)-6 and (20]. In addition, their low immunogenicity-due to their lack of expression of class II major histocompatibility complex and costimulatory molecules-makes MSCs able to escape alloantigen recognition and then unable to 
activate alloreactive $\mathrm{T}$ cells. The third important property of MSCs is their capacity to migrate and home to their target tissues after infusion, like other stem cells. There is a hypothesis that they can home into, and adjust their differentiation pathway to, diverse tissue microenvironments [21]. Animal studies have evaluated that MSCs can migrate into injured spinal cord tissue through cerebrospinal fluid and into the brain though peripheral blood [15, 22]. These properties make MSCs therapeutic potential cells in many chronic inflammatory demyelinating diseases and other central nervous system (CNS) diseases. MSCs have so far been applied successfully in patients with multiple sclerosis, neuromyelitis optica, and ischemic stroke, and in animal models of autoimmune encephalomyelitis [23-27]. In our center, UC MSCs have shown an extraordinarily therapeutic effect in patients with refractory lupus, inflammatory bowel disease, and rheumatoid arthritis [28-31]. All of these data suggest that MSCs may be exerted in the treatment of radiation myelitis.

\section{Method}

A 37-year-old male was diagnosed with laryngeal carcinoma, underwent total laryngectomy, and subsequently received more than 30 fractions of focal area radiotherapy in April 2010, with total radiation doses of 6000 cGy. One year later, the patient complained of right arm numbness and weakness, which progressively extended to both upper extremities and the area below his chest. Magnetic resonance imaging (MRI) of the spine demonstrated an enhanced lesion in the radiation field. Cytology of cerebrospinal fluid was normal. Four months after onset of clinical manifestations, leg weakness worsened resulting in multiple falls and difficulty in walking. Radiation myelitis was considered. Administration of corticosteroids (dexamethasone $10 \mathrm{mg} /$ day for 10 days) in local hospitals partially ameliorated the symptoms. However, 1 month later the patient suffered progressive worsening of lower limb numbness and weakness, and additional neurological symptoms such as absence of bladder sensation and incontinence emerged during tapering to low-dose steroids (prednisone $10 \mathrm{mg} /$ day). During the second period in the local hospital, treatment with the same dose of dexamethasone for 2 weeks, ganglioside, and mecobalamine did not show any beneficial results. The patient was then transferred to our department for further treatment.

Examination showed reduced power in four extremities ( 1 out of 5 on the Medical Research Council scale). Pain and temperature sensations were lost below the T2 level, while vibration and position sense were preserved. The remainder of the patient's general and neurologic examination was normal. The administration of corticosteroid (methylprednisolone $500 \mathrm{mg}$ /day intravenous pulse therapy) and mecobalamine was started, and omeprazole was also added. However, there were no improvements in muscle strength and bladder function after 5 days of high-dose methylprednisolone, so the dose was tapered based on the underlying adverse effects and ineffectiveness of steroids. A regimen of intravenous methylprednisolone $40 \mathrm{mg} /$ day together with omeprazole, aspirin, and mecobalamine was given on the following days. At the end of second week of admission, an MRI scan of the head and neck was taken. The craniocerebral scan was normal, while radiation myelopathy and radiation osteomyelitis was obvious (Fig. 1a): hyperintense signals were visible within the swollen cord from $\mathrm{C} 4$ to $\mathrm{C} 6$, with abnormal signal intensity in the vertebral body starting from $\mathrm{C} 2$ to T4. We thus turned to umbilical cord-derived mesenchymal stem cell transplantation (UC-MSCT), due to the unresponsiveness of steroid treatment and promising results for MSC transplantation in the treatment of CNS disorders.

UC MSCs were prepared by the Stem Cell Center of Jiangsu Province (Jiangsu Beike Bio-Technology, Taizhou, Jiangsu, China) as described previously with some modification [32, 33]. Fresh UC was obtained from an informed healthy mother in a local maternity hospital after normal delivery. First, a $10 \mathrm{ml}$ sample of cord blood was analyzed for communicable diseases, including hepatitis B virus, hepatitis $\mathrm{C}$ virus, HIV, cytomegalovirus, and syphilis. The UC was then rinsed twice, and the cord blood was removed during this process. The washed cords were cut into $1 \mathrm{~mm}^{2}$ pieces and floated in low-glucose Dulbecco's modified Eagle's medium (DMEM) containing $10 \%$ fetal bovine serum (FBS; Stemcell, Vancouver, Canada). The pieces of cord were subsequently incubated at $37{ }^{\circ} \mathrm{C}$ in a humidified atmosphere consisting of $5 \% \mathrm{CO}_{2}$. Nonadherent cells were removed by washing. The medium was replaced every 3 days after the initial plating. When welldeveloped colonies of fibroblast-like cells appeared after 10 days, the cultures were harvested with $0.05 \%$ trypsin/ ethylenediamine tetraacetic acid (Gibco Life Technologies, Grand Island, NY, USA) and passaged into a new flask for further expansion. UC MSCs used for treatment were subject to passing quality control tests, including immunophenotype identification and analysis of differentiation capacity. Flow cytometric analysis confirmed the cells expressed CD106, CD105, CD90, CD71, CD44, and CD29, but not CD34, CD14, CD3, or CD45 (all of the antibodies and their corresponding isotypes were purchased from $\mathrm{BD}$ Biosciences, San Jose, CA, USA). The capacity of MSCs to differentiate along adipogenic and osteogenic lineages was evaluated as described previously [32]. The cells with purity of more than $95 \%$ at passages 3 were used. Before transplantation, the cells were washed and the FBS was removed from the culture medium. For the procedure, a total of $5.2 \times 10^{7}$ cells suspended in $100 \mathrm{ml}$ saline were slowly infused by a heparinized syringe through the cubital vein of the arm over 30 minutes and $1.1 \times 10^{7}$ cells 

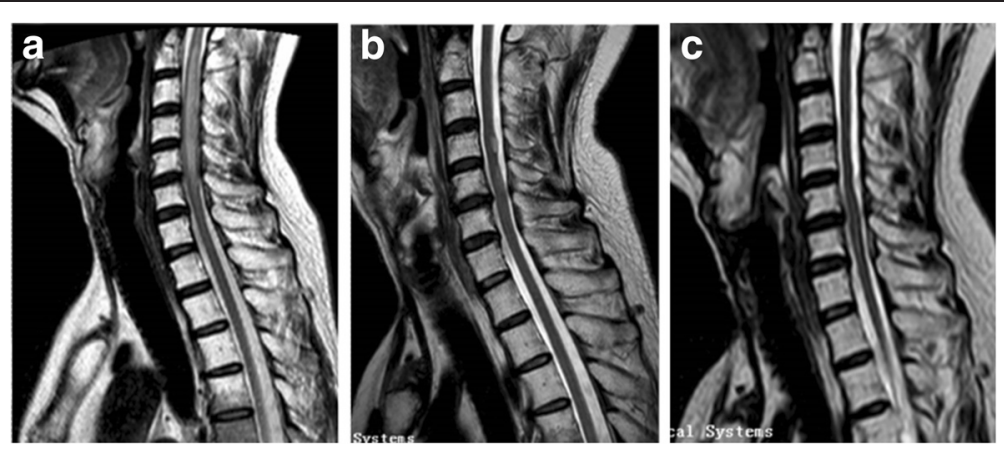

Fig. 1 Comparison of MRI T2-weighted images of the spinal cord. a MRI before UC-MSCT revealing fresh linear hyperintensities within the swollen cervical cord from C4 to C6. b At 6-month follow-up, MRI demonstrated obvious regression of the previous lesions. Swelling of the spinal cord was reduced significantly. c At 18-month follow-up, MRI did not show new enhanced lesions in the spinal cord

suspended in $10 \mathrm{ml}$ saline were slowly injected intrathecally, after informed consent was obtained from the patient and his family.

\section{Results}

No adverse events associated with transplantation were observed during or immediately after UC-MSCT in the patient.

\section{Two days post transplantation}

Two days after the transplantation, the patient presented less leg numbness and regained muscle strength of the four limbs against gravity. Bladder function improved 2 weeks after the transplantation. The patient was then even able to walk slowly with assistance and he was discharged from hospital in a stable condition with a regimen of prednisone $10 \mathrm{mg} /$ day and the neural nutrition medicine.

\section{One month post transplantation}

At 1-month follow-up after the transplantation, the patient displayed good general condition with leg numbness only. He was able to walk without assistance and his incontinence was replaced by urgency. Control MRI, taken 6 months later after transplantation, showed obvious regression of the previous enhancing lesions. The swelling of the spinal cord was reduced significantly (Fig. 1b).

\section{Nine months post transplantation}

Nine months after first discharge, the patient was readmitted to our center for second progressive leg weakness. He noted rapidly progressive difficulty in rising from low chairs and climbing stairs. Repeated MRI did not reveal a new enhancing lesion involving the spinal cord. He received the same therapy with UC-MSCT, combined with oral prednisone $20 \mathrm{mg} /$ day. The UC was obtained from another healthy mother. Therapy was still well tolerated and his symptoms returned to baseline.

\section{Eighteen months post transplantation}

The patient had been followed up for 18 months since the first transplantation. No severe infections, no local recurrence or distant metastasis of prior tumor, and no second tumor occurred. A follow-up MRI scan did not show new enhanced lesions in the spinal cord (Fig. 1c). In our case, neurological symptoms of the patient developed progressively with middle-dose steroid treatment, and did not improve after 5 days of high-dose steroid administration, so UC-MSCT was then employed. The improvement of the clinical and radiographic results suggested that UC-MSCT might be effective in patients with radiation myelitis. To our knowledge, this is the first report about UC-MSCT in treatment for radiation myelitis.

\section{Discussion}

Theories related to the pathogenesis of radiation-induced CNS lesions fall into two main categories [34, 35]. One category is necrosis due to the death or reproductive sterilization of glial cells and axons, while the other is vascular lesions such as edema, fibrin exudation, and even thrombosis and hemorrhage. Human autopsy studies showed different degrees of nonspecific inflammatory responses, including focal or diffuse infiltration of lymphocytic infiltration, reactive astrocytosis and microgliosis in injured spinal cord. These responses produce and release proinflammatory cytokines such as IL-1 and tumor necrosis factor alpha (TNFo) $[36,37]$. In our case, the improvement of neurological signs and symptoms was associated with or resulting from UC-MSCT, since treatment with steroids before the transplantation was ineffective. It is difficult to ascertain the direct role of MSC transplantation in the treatment of radiation myelitis. We hypothesize that the possible mechanistic rationale was mainly based on the abilities of MSCs to promote remyelination, to inhibit toxic inflammation, and to regenerate axons and neurons after they migrate into the lesion site, 
which requires further clinical and basic investigations for confirmation.

\section{Conclusion}

In summary, this case showed that MSC transplantation might be effective in treatment of radiation myelitis, although the length of follow-up was short. More clinical studies are needed to confirm the efficacy.

\section{Abbreviations}

CNS: Central nervous system; IL: Interleukin; MRI: Magnetic resonance imaging; MSC: Mesenchymal stem cell; NK: Natural killer; TNFa: tumor necrosis factor alpha; UC: Umbilical cord; UC-MSCT: umbilical cord-derived mesenchymal stem cell transplantation.

\section{Competing interests}

The authors have not been influenced by any financial or personal relationship with people or organizations in preparation of this report.

\section{Authors' contributions}

The work presented here was carried out in collaboration between all authors. LYS designed the study. DDW, HYZ, SYW, and CZ performed the transplantation. JL, FW, DDW, HYZ, SYW, and CZ collected the data and drafted the manuscript. LYS also participated in the drafting of the manuscript. All authors read and approved the final manuscript.

\section{Acknowledgements}

This study was supported by grants from the National Natural Science Foundation of China (No. 81571586, No. 81202333, No. 81471533, and No. 81302559)

Received: 30 June 2015 Revised: 20 August 2015 Accepted: 14 October 2015 Published online: 04 November 2015

\section{References}

1. Jeremic B, Djuric L, Mijatovic L. Incidence of radiation myelitis of the cervical spinal cord at doses of 5500 cGy or greater. Cancer. 1991;68:2138-41.

2. Lee AW, Kwong DL, Leung SF, Tung SY, Sze WM. Factors affecting risk of symptomatic temporal lobe necrosis: significance of fractional dose and treatment time. Int J Radiat Oncol Biol Phys. 2002;53:75-85.

3. Genc M, Genc E, Genc BO, Kiresi DA. Significant response of radiation induced CNS toxicity to high dose steroid administration. Br J Radiol. 2006;79:e196-9.

4. Shaw PJ, Bates D. Conservative treatment of delayed cerebral radiation necrosis. J Neurol Neurosurg Psychiatry. 1984;47:1338-41.

5. Goldwein JW. Radiation myelopathy: a review. Med Pediatr Oncol. 1987;15:89-95.

6. Bloss JD, DiSaia PJ, Mannel RS, Hyden EC, Manetta A. Radiation myelitis: a complication of concurrent cisplatin and 5-fluorouracil chemotherapy with extended field radiotherapy for carcinoma of the uterine cervix. Gynecol Oncol. 1991:43:305-7.

7. Jiang $Y$, Jahagirdar BN, Reinhardt RL, Schwartz RE, Keene CD. Pluripotency of mesenchymal stem cells derived from adult marrow. Nature. 2002:418:41-9.

8. Paul D, Samuel SM, Maulik N. Mesenchymal stem cell: present challenges and prospective cellular cardiomyoplasty approaches for myocardial regeneration. Antioxid Redox Signal. 2009;11:1841-55.

9. Ju S, Teng GJ, Lu H, Jin J, Zhang Y. In vivo differentiation of magnetically labeled mesenchymal stem cells into hepatocytes for cell therapy to repair damaged liver. Invest Radiol. 2010;45:625-33.

10. Bianco P, Riminucci M, Gronthos S, Robey PG. Bone marrow stromal stem cells: nature, biology, and potential applications. Stem Cells. 2001;19:180-92.

11. Dennis JE, Charbord P. Origin and differentiation of human and murine stroma. Stem Cells. 2002;20:205-14.

12. Pacary $E$, Legros $H$, Valable $S$, Duchatelle $P$, Lecoca M. Synergistic effects of $\mathrm{CoCl} 2$ and ROCK inhibition on mesenchymal stem cell differentiation into neuron-like cells. J Cell Sci. 2006:119:2667-78.

13. Wislet-Gendebien S, Hans G, Leprince P, Rigo JM, Moonen G. Plasticity of cultured mesenchymal stem cells: switch from nestin-positive to excitable neuron-like phenotype. Stem Cells. 2005;23:392-402.
14. Hou L, Cao H, Wang D, Wei G, Bai C. Induction of umbilical cord blood mesenchymal stem cells into neuron-like cells in vitro. Int J Hematol. 2003;78:256-61.

15. Satake K, Lou J, Lenke LG. Migration of mesenchymal stem cells through cerebrospinal fluid into injured spinal cord tissue. Spine. 2004;29:1971-9.

16. Liu J, Chen J, Liu B, Yang C, Xie D. Acellular spinal cord scaffold seeded with mesenchymal stem cells promotes long-distance axonregeneration and functional recovery in spinal cord injured rats. J Neurol Sci. 2013;325:127-36.

17. Nauta AJ, Fibbe WE. Immunomodulatory properties of mesenchymal stromal cells. Blood. 2007;110:3499-506.

18. Jiang XX, Zhang Y, Liu B, Zhang SX, Wu Y. Human mesenchymal stem cells inhibit differentiation and function of monocyte-derived dendritic cells. Blood. 2005;105:4120-6.

19. Sotiropoulou PA, Perez SA, Gritzapis AD, Baxevanis CN, Papamichail M. Interactions between human mesenchymal stem cells and natural killer cells. Stem Cells. 2006;24:74-85.

20. Uccelli A, Moretta L, Pistoia V. Mesenchymal stem cells in health and disease. Nat Rev Immunol. 2008:8:726-36.

21. Liechty KW, MacKenzie TC, Shaaban AF, Radu A, Moseley AM. Human mesenchymal stem cells engraft and demonstrate site-specific differentiation after in utero transplantation in sheep. Nat Med. 2000;6:1282-6.

22. Chen J, Li Y, Wang L, Zhang Z, Lu D. Therapeutic benefit of intravenous administration of bone marrow stromal cells after cerebral ischemia in rats. Stroke. 2001;32:1005-11.

23. Bang OY, Lee JS, Lee PH, Lee G. Autologous mesenchymal stem cell transplantation in stroke patients. Ann Neurol. 2005:57:874-82.

24. Koh SH, Kim KS, Choi MR, Jung KH, Park KS. Implantation of human umbilical cord-derived mesenchymal stem cells as a neuroprotective therapy for ischemic stroke in rats. Brain Res. 2008;1229:233-48.

25. Siatskas C, Payne N, Short M, Bernard CA. A consensus statement addressing mesenchymal stem cell transplantation for multiple sclerosis: it's time! Stem Cell Rev Rep. 2010;6:500-6.

26. Lu Z, Ye D, Qian L, Zhu L, Wang C. Human umbilical cord mesenchymal stem cell therapy on neuromyelitis optica. Curr Neurovasc Res. 2012;9:250-5.

27. Fisher-Shoval Y, Barhum Y, Sadan O, Yust-Katz S, Ben-Zur T. Transplantation of placenta-derived mesenchymal stem cells in the EAE mouse model of MS. J Mol Neurosci. 2012;48:176-84.

28. Sun L, Wang D, Liang J, Zhang H, Feng X. Umbilical cord mesenchymal stem cell transplantation in severe and refractory systemic lupus erythematosus. Arthritis Rheum. 2010;62:2467-75.

29. Li X, Wang D, Liang J, Zhang H, Sun L. Mesenchymal SCT ameliorates refractory cytopenia in patients with systemic lupus erythematosus. Bone Marrow Transplant. 2013:48:544-50.

30. Liang J, Zhang $H$, Wang D, Feng $X$, Wang H. Allogeneic mesenchymal stem cell transplantation in seven patients with refractory inflammatory bowel disease. Gut. 2012;61:468-9.

31. Liang J, Li X, Zhang H, Wang D, Feng X. Allogeneic mesenchymal stem cells transplantation in patients with refractory RA. Clin Rheumatol. 2012;31:157-61.

32. Lu LL, Liu YJ, Yang SG, Zhao QJ, Wang X. Isolation and characterization of human umbilical cord mesenchymal stem cells with hematopoiesissupportive function and other potentials. Haematologica. 2006;91:1017-26.

33. Chen G, Yue A, Ruan Z, Yin Y, Wang R. Human umbilical cord-derived mesenchymal stem cells do not undergo malignant transformation during long-term culturing in serum-free medium. PLoS One. 2014;9:e98565.

34. Schultheiss TE, Stephens LC, Maor MH. Analysis of the histopathology of radiation myelopathy. Int J Radiat Oncol Biol Phys. 1988;14:27-32.

35. Shirazi A, Ghobadi G, Ghazi-Khansari M. A radiobiological review on melatonin: a novel radioprotector. J Radiat Res. 2007:48:263-72.

36. Schultheiss TE, Stephens LC. Permanent radiation myelopathy. Br J Radiol. 1992;65:737-53.

37. Nordal RA, Wong CS. Molecular targets in radiation-induced blood-brain barrier disruption. Int J Radiat Oncol Biol Phys. 2005;62:279-87. 